SPhT/93-004(Revised)

\title{
Dimensionality of spacetime as a gauge-invariance parameter in Yang-Mills calculations
}

\author{
Rajesh R. Parwani \\ Service de Physique Théorique, CE-Saclay \\ 91191 Gif-sur-Yvette, France. \\ PACS 11.15Bt, 12.38Bx, 12.38Qk. \\ 1 February 1993 \\ Revised April 21, 1993
}

\begin{abstract}
It is described how the dimensionality of space-time may be used to check the gauge invariance of perturbative calculations in pure Yang-Mills $(Y M)$ theories. The idea is based on the fact that pure $Y M$ theory in two dimensions is perturbatively free. Thus gauge-invariant quantities evaluated in a $D$ dimensional pure $Y M$ theory should vanish as $D$ goes to two. The procedure and various subtleties in its application are illustrated by examples drawn from quarkless $Q C D$ at zero and nonzero temperature. The inclusion of quarks and the use of background field gauges is briefly discussed.
\end{abstract}

*email : parwani@wasa.saclay. cea.fr 


\section{Introduction}

Perturbative calculations of gauge invariant quantities necessarily proceed in a gauge noninvariant manner due to the gauge-fixing required in the Lagrangian. In order to verify the gauge-invariance of the final result, and to check against possible errors, computations are usually repeated for different choices of the gauge-fixing or they are performed in a general class of gauges labelled by an arbitrary gauge-fixing parameter. In the latter case, one ascertains that the dependence on the gauge-parameter drops out for physical quantities. For Yang-Mills $(Y M)$ theories, the complicated tensor structure of the vertices makes calculations in a general gauge containing a gauge parameter extremely tedious. In this paper I describe how, for pure $Y M$ theories, one may perform calculations in any particular gauge with a convenient propagator (e.g. Feynman) and yet retain a nontrivial check on the gauge-invariance of the result.

The idea uses the fact that pure YM theory in two dimensional space-time is perturbatively free. This is established by going to the axial $\left(A_{1}=0\right)$ gauge whence the gauge self-interactions vanish. Since, by definition, gauge-invariant quantities are independent of the choice of gauge-fixing, all gauge-invariant quantities in pure $Y M_{2}$ theory must vanish. The strategy to use this fact for calculating physical quantities in some $D_{0}$ dimensional space-time is as follows : perform the Lorentz algebra and loop integrals for an arbitrary $D$ dimensions; then if the quantity being calculated is truly gauge-invariant, a necessary condition is that it should vanish at $D=2$. In this way the dimensionality of space-time is used as a gauge-invariance parameter.

As will be seen later, for all but one example in this paper the $D$ dependence of the Lorentz algebra gives the sole useful check on gauge-invariance. However we will encounter an example of a gauge-invariant quantity whose only $D$ dependence is in the loop integral. In order to treat all possibilities in a unified manner it is necessary to adopt prescriptions for defining the $D \rightarrow 2$ limit in the integrals. Integrals like those from zero-temperature Feynman diagrams are defined in $D$ dimensions by analytic continuation [1, 2, 3, 国 with the $D \rightarrow 2$ limit taken after doing the integrals. For 
nonzero temperature integrals containing Bose-Einstein factors, an infrared cutoff will be imposed.

The reader is advised that it is not the aim of this paper to provide, in a single attempt, a perturbative analysis of pure $Y M$ theory for all $D_{0} \geq D \geq 2$ dimensions (if indeed such a thing is possible), but rather to define a pragmatic procedure that connects correctly calculated gauge-invariant quantities near $D=D_{0}$ to the value zero at $D=2$. The prescriptions are needed for the loop integrals because even if the gauge-invariant quantity is well defined near $D=D_{0}$, in the limit $D \rightarrow 2$ one will encounter infrared (IR) singularities symptomatic of lower dimensional field theories. Of course the prescriptions mentioned were chosen because they gave sensible results for the examples considered. They remain to be checked in other cases as the author has no general proof of their validity. Note that for zero temperature type of integrals dimensional continuation is being used here to extrapolate gauge invariant quantities from $D=D_{0}$ down to $D=2$, in contrast to its usual role of regulating ultraviolet (UV) and IR [1, 2, 3, 4] singularities near $D=D_{0}$.

The $D \rightarrow 2$ check described above cannot be used for gauge-invariant quantities which are dimension specific. An example is the perturbative beta function of $D_{0}=4 Y M$ theory which gives information about the UV behaviour of Green's functions. Within mass-independent renormalisation schemes, the beta function is scheme-independent up to second order and is manifestly gauge independent when minimal subtraction is used [3, 4]. It is a dimension specific quantity because it is obtained from the residue of the pole, as $D \rightarrow 4$, of the coupling constant renormalisation factor. $Y M$ theory is super renormalisable for $D<4$ and therefore lacks the conventional UV beta function. It is thus not apparent if one may sensibly extrapolate the conventional beta function beyond an infinitesimal range near $D=4$. More examples of dimension specific gauge invariant quantities may be found in $D_{0}=3$ pure $Y M$ theory with an added Chern-Simons term [5]. The Chern-Simons term is specific to odd dimensions and so here again one does not, in general, expect gauge-invariant 
quantities to vanish as $D \rightarrow 2$.

The nice thing about performing the Lorentz algebra in $D$ dimensions (in addition to the integrals) is that it takes almost no more effort than in doing it for the physical $D_{0}$ dimensions. The benefit, as mentioned above, is that the $D$ parameter used in a simple gauge provides one with an algebraically efficient way of checking gauge invariance. Of course, one may use the $D$ parameter in conjunction with a conventional gauge parameter $(\alpha)$ to give additional checks and insight. The $D$ parameter is a book-keeping device keeping track of the "relevant" $(D-2)$ pieces in a calculation while the $\alpha$ parameter prefaces the "irrelevant" pieces.

What about fermions? Clearly $Q C D_{2}$ with fermions is a nontrivial theory [6]. Fortunately, the contribution of fermions to amplitudes can be kept track of by using the usual trick of working with an arbitrary $N_{f}$ copies of them. A gauge-invariant quantity must be separately gauge invariant in the $N_{f}=0$ and $N_{f} \neq 0$ sectors. In the first sector, the calculations may be performed as described above using the $D$ parameter to check gauge-invariance while the $N_{f} \neq 0$ sector can be analysed separately. Usually diagrams with one or more fermion lines are algebraically simpler to deal with than those with only gluon lines so the methodology described here is not without promise.

The idea outlined in the preceding paragraphs will be exemplified in this paper for zero and nonzero temperature $(T=1 / \beta)$ pure $Y M$ theory with gauge group $S U\left(N_{c}\right)$ at $D_{0}=4$. In Sect.(2) gluon-gluon scattering at zero temperature is considered at tree level. This is a relatively simple example since there are no loop integrals to complicate matters. The metric used in Sect.(2) is Minkowskian, $\operatorname{diag}\left(g_{\mu \nu}\right)=(1,-1, \ldots .,-1)$. In Sects.(3-5) the examples are at nonzero $T$ and the metric is Euclidean, $g_{\mu \nu}=\delta_{\mu \nu}$ (for orientation to nonzero temperature field theory see, for example, [4, \&]). The measure for loop integrals in Sects.(3-5) is

$$
\int[d q] \equiv T \sum_{q_{0}} \int \frac{d^{(D-1)} q}{(2 \pi)^{(D-1)}},
$$


where the sum is over discrete Matsubara frequencies [7, 8], $q_{0}=2 \pi n T$ for gauge bosons and ghosts, $n \in \mathcal{Z}$. For quantities which depend on the external momenta, an analytic continuation to Minkowski space is made as usual after the loop sums are done [8]. In Sect.(3) the one-loop gluon self-energy is considered and the two prescriptions for loop-integrals are introduced while in Sect.(4) a discussion is given of "hard thermal loops" and propagator poles in $D$ dimensions. Sect.(5) considers the free energy of a gluon plasma to third order. The "plasmon" contribution in $D$ dimensions requires the simultaneous use of both prescriptions introduced in Sect.(3), therefore providing a check on their consistency. The conclusion is in Sect.(6) while the Appendix contains some expressions and discussion mentioned in the main text.

The following gauges will be frequently referred to throughout the paper : the strict Coulomb gauge $\left(\xi=0\right.$ limit of the $(\nabla \cdot \vec{A})^{2} / 2 \xi$ gauge- fixing), the $\alpha$-covariant gauge with gauge-fixing term $\left(\partial_{\mu} A_{\mu}\right)^{2} / 2(\alpha+1)$ and the Feynman gauge $(\alpha=0)$. The Feynman rules, being standard [3, 4, 7, 8], will not be spelled out. $D$ - vectors will be denoted by uppercase and have Greek indices, $Q_{\mu}=\left(q_{0}, \vec{q}\right), q \equiv|\vec{q}|$, and the $(D-1)$ spatial components will be labelled by Roman letters $(i, j)$. Keep in mind that in $D$ dimensions the coupling $g^{2}$ has a mass dimension $(4-D)$.

\section{Gluon-gluon scattering}

The scaterring amplitude $M(g g \rightarrow g g)$, for two gluons into two gluons, involves at lowest order four Feynman diagrams [9]. The first comes from the order $g^{2}$ fourpoint vertex in the Lagrangian while the other three are formed from two three-point vertices tied by a propagator and represent the usual $s, t$ and $u$ channel scatterings. The sum of the four amputated Feynman diagrams gives the tensor $T_{\mu \nu \sigma \tau}$, where the Lorentz indices indicate the external gluon legs. The gauge-invariant amplitude is then given by 


$$
M=T_{\mu \nu \sigma \tau} \epsilon_{1}^{\mu} \epsilon_{2}^{\nu} \epsilon_{3}^{\sigma} \epsilon_{4}^{\tau}
$$

Here $\epsilon_{(n)}^{\mu} \equiv \epsilon^{\mu}\left(\vec{k}, \lambda_{(n)}\right)$ represents the polarisation vector for the $n$-th $(n=1,2,3,4)$ gluon with physical polarisation $\lambda_{(n)}$ and on-shell momentum $K^{2}=k_{0}^{2}-\vec{k}^{2}=$ $K^{\mu} \epsilon_{\mu}(\vec{k}, \lambda)=0$. In practice one usually needs the squared amplitude summed over initial and final spin (and colour) variables. Choosing the basis $\epsilon_{\mu}(\vec{k}, \lambda) \equiv(0, \vec{\epsilon})$, one has the transverse projection operator

$$
P_{\mu \nu}(K)=\sum_{\lambda} \epsilon_{\mu}(\vec{k}, \lambda) \epsilon_{\nu}(\vec{k}, \lambda)=\left(\delta_{i j}-\frac{k_{i} k_{j}}{k^{2}}\right) \delta_{\mu i} \delta_{\nu j}
$$

When the relation (2.2), which is true in any dimension, is used to evaluate $\sum_{\lambda}|M|^{2}$ in $D$ dimensions, factors of $D$ will appear. For example $g^{\mu \nu} P_{\mu \nu}=(D-2)$ and so in particular $P_{\mu \nu}=0$ in two dimensions because then there are no transverse states. From Ref. 10 one obtains

$$
\Sigma_{\text {spin,colour }}|M(g g \rightarrow g g)|^{2}=4 g^{4} N_{c}^{2}\left(N_{c}^{2}-1\right)(D-2)^{2}\left[3-\frac{u t}{s^{2}}-\frac{u s}{t^{2}}-\frac{s t}{u^{2}}\right] .
$$

For $D=4$ this reduces to earlier results [9] and it also vanishes when $D \rightarrow 2$ as desired. However there are two subtleties which should be noted. Firstly, since the on-shell gluons are massless, there are kinematic singularities in (2.3) even for $D \neq 2$ : for example, $s=\left(K_{1}^{\mu}+K_{2}^{\mu}\right)^{2}=0$ when $\overrightarrow{k_{1}}$ is parallel to $\overrightarrow{k_{2}}$. As $D \rightarrow 2$, the Mandelstam variables $(s, t, u)$ vanish when the vectors $\left(\overrightarrow{k_{2}}, \overrightarrow{k_{3}}, \overrightarrow{k_{4}}\right)$ are respectively in the same direction as $\overrightarrow{k_{1}}$. Thus the $D \rightarrow 2$ limit of (2.3) is unambiguous only if the kinematic singularities are regulated. Secondly, if one also averages over initial spins in $D$ dimensions, then $(2.3)$ is divided by $(D-2)$ for each of the incoming lines. This averaging is fine if one is working near $D=4$ say 110 , but is clearly inadvisable if one wants to check gauge-invariance by the $D \rightarrow 2$ procedure : In the $D \rightarrow 2$ method one should check gauge-invariant quantities before performing other 
extraneous $D$-dependent operations.

\section{Self-energy}

The self-energy by itself is not a gauge-invariant quantity. However at nonzero temperature there is a gauge-invariant piece of it which is easy to extract at low orders. This is the inverse screening length for static electric fields, also called the electric

mass, $m_{e l}$. If $\delta^{a b} \Pi_{\mu \nu}\left(k_{0}, \vec{k}\right)$ is the gluon polarisation tensor at nonzero temperature, then at lowest order one may define

$$
m_{e l}^{2} \equiv-\Pi_{00}(0, \vec{k} \rightarrow 0)
$$

At $D_{0}=4$, the order $(g T)^{2}$ result for (3.1) is well known [8, 11]. Remarkably it was found in Ref. 12 that the next term of order $g^{2}|\vec{k}| T$ in the low momentum expansion of $\Pi_{00}(0, \vec{k})$ at one-loop is independent of the $\alpha$ parameter in the $\alpha$-covariant gauge and also has the same value in the Coulomb gauge, thus suggesting that even this term is gauge-invariant. I repeat here the analysis of Ref.[12] using the $D$ parameter. In the $\alpha$-covariant gauge one finds for the sum of one-loop gluonic and ghost diagrams, the relevant object

$$
\Pi_{00}(0, \vec{k})=\frac{g^{2} N_{c}}{2}\left[A_{0}(\vec{k})+\alpha A_{1}(\vec{k})+\alpha^{2} A_{2}(\vec{k})\right]
$$

where

$$
A_{0}(\vec{k})=\int[d q] \frac{2(D-2)\left(2 q_{0}^{2}-Q^{2}\right)+4 k^{2}}{Q^{2}\left[q_{0}^{2}+(\vec{q}-\vec{k})^{2}\right]}
$$




$$
\begin{aligned}
& A_{1}(\vec{k})=\int[d q] \frac{2\left[4(\vec{k} \cdot \vec{q})^{2}-2 k^{2} Q^{2}+2 q_{0}^{2} k^{2}\right]}{Q^{4}\left[q_{0}^{2}+(\vec{q}-\vec{k})^{2}\right]} \\
& A_{2}(\vec{k})=\int[d q] \frac{q_{0}^{2} k^{4}}{Q^{4}\left[q_{0}^{2}+(\vec{q}-\vec{k})^{2}\right]^{2}} .
\end{aligned}
$$

The only difference between the integrands in eqns.(3.2 - 3.5) and the expressions studied in [12, 13] is the presence of the factor $(D-2)$, coming from the Lorentz algebra, in eq.(3.3). This factor is invisible in [12, 13] because they work with $D=$ $D_{0}=4$. From the above expressions, one gets for the electric mass squared at order $g^{2}:$

$$
m_{e l}^{2}=g^{2} N_{c}(D-2) \int[d q] \frac{\left(2 q^{2}-Q^{2}\right)}{Q^{4}} .
$$

After performing the frequency sum and angular integrals one obtains

$$
m_{e l}^{2}=g^{2} N_{c}(D-2) T^{(D-2)} \omega(D)[2 J(D)-I(D)],
$$

where

$$
\begin{aligned}
\frac{1}{\omega(D)} & =2^{(D-2)} \pi^{\left(\frac{D-1}{2}\right)} \Gamma\left(\frac{D-1}{2}\right), \\
I(D) & =\int_{0}^{\infty} d x x^{(D-3)} n_{x}, \\
J(D) & =\frac{1}{2} \int_{0}^{\infty} d x x^{(D-3)}\left[n_{x}-x \frac{d}{d x} n_{x}\right], \\
n_{x} & =1 /\left(e^{x}-1\right) .
\end{aligned}
$$

Both of the integrals $I(D)$ and $J(D)$ are IR finite for $D>3$. The second term in $J(D)$ may be integrated by parts, and the surface term dropped when $D>3$, resulting in

$$
J(D)=\frac{1}{2}(D-1) I(D) \quad, D>3 .
$$


The integral $I$ can be written in terms of gamma and zeta functions [14

$$
I(D)=\Gamma(D-2) \zeta(D-2) \quad, D>3
$$

Thus one may write eq. (3.7) as

$$
m_{e l}^{2}=g^{2} N_{c}(D-2) T^{(D-2)} \omega(D) \Gamma(D-1) \zeta(D-2) \quad, D>3 .
$$

The divergence as $D \rightarrow 3$ shows up in the zeta-function. In a consistent calculation at $D_{0}=3$, the logarithmic divergence in the naive expression for $m_{e l}$ will be cutoff by $g^{2} / T$ 15. Suppose one continues (3.14) down to $D=2$. Then the result vanishes because of the $(D-2)$ Lorentz factor. However this may be fortuitous as it is related to the possibility of simplifying $J(D)(3.10)$ through an integration by parts, dropping a surface term, and getting a result proportional to $I(D)$, so that the square brackets in (3.7) has no net singularity at $D=2$. In more complicated examples one may not be so lucky. Therefore a prescription will now be introduced to handle the IR singularities in integrals like $I(D)$ and $J(D)$ above. It is simply this : integrals with Bose-Einstein factors will be interpreted for $D \leq 3$ with an infrared cutoff $\lambda$ :

$$
\int_{0}^{\infty} \rightarrow \int_{\lambda}^{\infty}
$$

That is, the lowest order electric mass is given in $D>3$ dimensions by the expressions (3.14) and is defined, for the purpose of this paper, by (3.7 - 3.11, 3.15) in $D \leq 3$ dimensions. The cutoff in (3.15) is left unspecified since it is required here only to allow the limit $D \rightarrow 2$ to be taken with impunity. If one is really interested in the problem in $D_{0} \leq 3$ dimensions then the cutoff must be determined self- consistently. In this paper the interest is in gauge-invariant quantities near $D_{0}=4$ and the prescription (3.15) allows the connection to be made with the free theory at $D=2$. The prescription (3.15) will be tested in Sects. $(4,5)$.

Now consider the order $|\vec{k}| T$ term in (3.2) for $D=4$. As discussed in [12, this can only arise from the infrared region of the integrals. That is, it only arises from the $q_{0}=0$ part of the frequency sum (1.1) in (3.3, 3.4). For the gauge-fixing 
dependent piece (3.4), the zero mode contains pieces exactly of order $|\vec{k}| T$ but the net contribution vanishes after the elementary integrals are done [12]. The zero mode in the $\alpha$ independent piece $(3.3)$ contributes

$$
T \int \frac{d^{(D-1)} q}{(2 \pi)^{(D-1)}}\left[\frac{-2(D-2)}{(\vec{q}-\vec{k})^{2}}+\frac{4 k^{2}}{q^{2}(\vec{q}-\vec{k})^{2}}\right] .
$$

The first term in (3.16) vanishes by dimensional regularisation. The second gives, at $D=4$, the contribution proportional to $|\vec{k}| T$ found in 12 . In $D$ dimensions this last piece has no $(D-2)$ factor from the Lorentz algebra but the integral is highly singular for $D \leq 3$ even when $\vec{k} \neq 0$. As the integral is similar to that occurring in zero temperature field-theory (indeed (3.16) is a contribution in the effective $(D-1)$ dimensional Euclidean field theory which represents the far infrared, or infinite temperature, limit of the $D$ dimensional finite temperature field theory [8, 16].) it is natural to use dimensional continuation methods [1, 2, 3, 4] for its evaluation. A standard calculation of (3.16) yields,

$$
T\left(\frac{D-2}{4}\right) \frac{k^{(D-3)}}{(4 \sqrt{\pi})^{(D-4)}} \frac{1}{\Gamma(D / 2) \cos (\pi D / 2)} .
$$

Amazingly, $D=2$ is the only positive value of $D$ for which (3.17) vanishes. Thus the $(k T)$ term in (3.16) at $D=4$ does satisfy the necessary condition for gaugeinvariance once the integral is defined by dimensional continuation for the $D \rightarrow 2$ limit. Of course the above analysis does not explain why the $k T$ term is gaugeinvariant. In Ref.[12] it was related to a higher order term in the free energy but its direct physical significance is unclear to the present author. It might be interesting also to have a general proof for the gauge-invariance of the $k T$ term using, for example, the techniques of Ref.[17].

The use of dimensional continuation to evaluate zero temperature type integrals is the second prescription that will be used in this paper. Another example of its use will be given in Sect.(5). Here it is noted that with the replacement $(D-1) \rightarrow D$, the second integral in (3.16) occurs in the zero temperature self-energy in $D$ dimensions. 
The zero-temperature self-energy thus diverges when $D \rightarrow 2$ (i.e. $D \rightarrow 3$ in (3.17)) but this is not worrisome since the self-energy is a gauge-dependent object.

\section{4 'Hard Thermal Loops' and propagator poles}

For $Q C D_{4}$, at nonzero temperature, there are an infinite number of bare loop diagrams which are as large as the tree amplitudes when the momentum entering the external legs is soft $(\sim g T)$ and the internal loop momentum is hard $(\sim T)$. These "hard thermal loops" (HTL) occur only at one-loop and have been extensively analysed by Braaten and Pisarski [18] and Frenkel and Taylor [19]. The HTL's exist for amplitudes when all the $N \geq 2$ external lines are gluons or when one pair is fermionic and the other $(N-2)$ are gluons. By explicit calculations [18, 19], the HTL's were found to be the same in Coulomb, $\alpha$-covariant and axial gauges. General proofs of gauge-fixing independence may be constructed [20]. A gauge-invariant generating functional for the HTL's that was constructed by Taylor and Wong has been cast into myriad forms [21]. In some recent work, Blaizot and Iancu 222 have rederived the results of [18, 19, 21] by analysing the kinetic equations obtained through a selfconsistent truncation of the Schwinger-Dyson equations for sources and fields at finite temperature.

From the expressions contained in [18] or [21, 22] one sees that the $N_{f}=0$ sector of the $N$-gluon HTL contains an overall factor of $(D-2)$ when the Lorentz algebra is done in $D$ dimensions. Even the HTL's with external quark lines are seen to be proportional to $(D-2)$. As noted in the above papers, this is because the HTL's, which are the leading high temperature (and essentially classical) parts of the one loop diagrams, receive contributions only from the $(D-2)$ physical transverse gluon degrees of freedom.

To consider the pure gluonic HTL's in $D$ dimensions (the $N_{f} \neq 0$ sector is not of interest here), the $D$ dependence of the integrals must also be taken into account 
(see also Frenkel and Taylor [21]). For the purpose of power counting it is convenient to introduce the dimensionless coupling $g_{0}$ in $D$ dimensions through the relation $g^{2}=g_{0}^{2} T^{(4-D)}$, where the temperature has been chosen as the mass scale since that is the natural parameter in the problem. Now a hard momenta is of order $T$ while soft refers to $\sim g_{0} T$. With this notation one can repeat all the relevant analysis of [18, 19, 21, 22] and show that it remains valid for $D>3$ dimensions. However naive power counting suggests that for $D \leq 3$ dimensions soft thermal loops (loop momenta $\left.\sim g_{0} T\right)$ are no longer suppresed relative to HTL's. This is related to the occurrence of IR divergences; for example, the static limit of the HTL in the gluon self-energy [23] is simply the electric mass squared (3.1) which was noted in the last section to diverge in the naive $D \rightarrow 3$ limit. Therefore, just as in the case of $m_{e l}$, for the purpose of taking the $D \rightarrow 2$ limit, HTL's are defined in this paper for $D \leq 3$ with the infrared cutoff (3.15). Then they vanish as $D \rightarrow 2$ simply because of the Lorentz algebraic factor.

Just as at zero temperature, the physical poles of the propagator at non-zero temperature are gauge invariant [20]. At nonzero temperature, the real part of the gauge propagator pole at zero external three momentum defines the induced thermal masses for the gluons and for $D_{0}=4$ the leading $(\sim g T)$ result is easily obtained at one-loop [8]. When using the $D$ parameter, the thermal mass will vanish near $D=2$ as $\sim \sqrt{(D-2)}$, just like the electric mass (3.1), when the prescription (3.15) is adopted. The imaginary part (at $D_{0}=4$ ) turns out to be of subleading order $\left(g^{2} T\right)$ and a practical consistent calculation requires the Braaten-Pisarski [18 resummation using propagators and vertices dressed with HTL's. If the calculation of the imaginary part is done in $D$ dimensions there will be three sources of $D$ dependence : from the HTL's in the effective propagators and vertices, from the Lorentz algebra of the dressed diagrams, and from the loop integral of the dressed diagrams. It would be interesting to see how the $D \rightarrow 2$ limit looks like in this case but this will not be attempted here because the analysis is tedious. In the next section an 
example will be considered which also involves a resummation but is easier to analyse.

\section{$5 \quad$ Free energy}

The free-energy is physical quantity equal to the negative of the pressure and is directly obtainable by calculating bubble diagrams in perturbation theory [24]. Since it is physical, it must be gauge-invariant. In the Feynman gauge, the ideal gas pressure $\left(P_{0}\right)$ of gluons is given by [7, 8]

$$
\begin{aligned}
\frac{P_{0} V}{T} & =\left(N_{c}^{2}-1\right) \ln \left\{\left[\operatorname{Det}\left(-\partial^{2} \delta_{\mu \nu}\right)\right]^{-\frac{1}{2}} \cdot \operatorname{Det}\left(-\partial^{2}\right)\right\} \\
& =(D-2)\left(N_{c}^{2}-1\right) \ln \left[\operatorname{Det}\left(-\partial^{2}\right)\right]^{-\frac{1}{2}},
\end{aligned}
$$

where $V$ is the volume. The first determinant in (5.1) is the contribution of gluons while the second determinant is the ghost contribution. The first two terms in (5.2) count the number of physical degrees of freedom. The remaining expression in (5.2) may be evaluated (see appendix) to yield the free gluonic pressure in $D$ dimensions,

$$
P_{0}=(D-2)\left(N_{c}^{2}-1\right) T^{D} \pi^{-\frac{D}{2}} \Gamma(D / 2) \zeta(D), D>1 .
$$

The result is positive for $D>2$ and vanishes smoothly in the limit $D \rightarrow 2$. The first singularity appears in the zeta-function at $D=1$ when field theory collapses to quantum mechanics.

Consider next the order $g^{2}$ correction to the ideal gas pressure, $P_{2}$. In the Feynman gauge one obtains after some algebra,

$$
\begin{aligned}
P_{2} & =g^{2} N_{c}\left(N_{c}^{2}-1\right)\left[\int \frac{[d q]}{Q^{2}}\right]^{2}\left\{-\frac{1}{2}\left(\frac{1}{2}\right)+\frac{1}{8}[2 D(1-D)]+\frac{1}{12}[9(D-1)]\right\} \\
& =-\left(\frac{D-2}{2}\right)^{2} g^{2} N_{c}\left(N_{c}^{2}-1\right)\left[\int \frac{[d q]}{Q^{2}}\right]^{2} \\
& =-\left(\frac{D-2}{2}\right)^{2} g^{2} N_{c}\left(N_{c}^{2}-1\right) T^{(2 D-4)} \omega^{2}(D) I^{2}(D) .
\end{aligned}
$$


The terms within brackets in (5.4) come respectively from the two-loop bubble diagrams with one, two and three gluon propagators. Shown explicitly in front of each contribution are the symmetry factors and the minus sign for the ghost loop. The functions $\omega(D)$ and $I(D)$ in (5.6) are those defined earlier in eqns.(3.8, 3.9). When $D>3$ one may also use eq.(3.13) and at $D=4$ one recovers a known result [24]. For $D<3$ the prescription (3.15) is again to be used for the integral $I(D)$. Then the net result in (5.6) vanishes for $D=2$ as required for a gauge invariant quantity. The main point here is that if one had made errors (for example in the symmetry factors in (5.4)), these would likely have shown up in the nonvanishing of the net result at $D=2$. A similar calculation in an $\alpha$-covariant gauge for the purposes of checking algebra is far more tedious, especially for the diagram with three gluon lines. The complexity of the algebra in an $\alpha$-gauge in fact increases the sources of possible errors at intermediate steps. As a curiosity, it might interest the reader to note that nevertheless the result (5.5) can also be established in an $\alpha$-covariant gauge before doing any explicit integrals, albeit with greater algebraic effort, the $\alpha$ dependence cancelling in the sum of diagrams as required (see appendix).

The next correction to the pressure in four dimensions is of order $g^{3}$. This "plasmon" correction is a nonperturbative contribution and it was computed in $Q C D$ by Kapusta [24, 25]. It is obtained by summing an infinite class of IR divergent diagrams, formed by adding two or more self-energy subdiagrams along the gluon line of the one-loop bubble diagram. The leading correction $\left(\sim g^{3}\right)$ is due to the electric mass, $\Pi_{00}(0, \vec{k} \rightarrow 0)$. Summing the electric mass insertions in $D$ dimensions gives

$$
P_{3}=-\frac{\left(N_{c}^{2}-1\right)}{2 \beta} \int \frac{d^{(D-1)} q}{(2 \pi)^{(D-1)}}\left[\ln \left(1+\frac{m_{e l}^{2}}{q^{2}}\right)-\frac{m_{e l}^{2}}{q^{2}}\right] .
$$

The above expression is well defined for $D>3$ with $m_{e l}$ given by (3.14). The loop 
integral may be evaluated using zero temperature techniques (see appendix) to give

$$
P_{3}=\frac{\left(N_{c}^{2}-1\right)}{2 \beta} \frac{\Gamma\left(\frac{1-D}{2}\right)\left(m_{e l}^{2}\right)^{\frac{D-1}{2}}}{(4 \pi)^{\frac{D-1}{2}}}, D>3 .
$$

Since $m_{e l} \sim g$, the result (5.8) is subleading, when $D>3$, to the order $g^{2}$ contribution $P_{2}$ given by eq.(5.6). Also note that (5.8) is positive for $3<D<5$ so that it opposes $P_{2}$ in that range. In order to apply the $D \rightarrow 2$ check on (5.7) we need to use both of the prescriptions introduced earlier. Firstly, for $D<3$ the electric mass $m_{e l}$ is defined by the cutoff prescription (3.7 3.11, 3.15). Secondly, the loop integral in (5.7) is IR divergent for $D<3$ and so it is defined by the analytic continuation prescription (the IR divergence coincides with a physical effect : the magnetic contribution may no longer be subleading (see appendix)). Thus one takes the $D \rightarrow 2$ limit in (5.8)

with $m_{e l}$ defined by $(3.7,3.15)$. Since $m_{e l} \sim \sqrt{(D-2)}$, therefore when $D \rightarrow 2, P_{3}$ vanishes as $\sim(D-2)^{\gamma}$ with $\gamma=\frac{1}{2}+\frac{(D-2)}{2}$. The $D$ dependent exponent $\gamma$ is another sign of the nonperturbative nature of the plasmon term. The nontrivial point here is that the loop integral has not introduced any adverse powers of $(D-2)$ which would have had a disastrous effect for the $D \rightarrow 2$ limit. This example shows a successful cohabitation of the two IR prescriptions that were introduced for defining the $D \rightarrow 2$ limit.

\section{Conclusion}

The dimensionality of spacetime $(D)$ has been proposed and illustrated as a possibly efficient and beneficial way to check gauge-invariance in pure YM theories. Gauge invariant quantities which are not dimension specific should vanish as $D \rightarrow 2$. The converse is not necessarily true. For example, any quantity, even if gauge variant, when calculated in the axial gauge should vanish as $D \rightarrow 2$ due to the free nature of pure $Y M_{2}$.

Although in most of the examples it was the Lorentz algebra which contained the useful $D$-dependent information, the procedure required the use of two ad hoc 
prescriptions to define the $D \rightarrow 2$ limit in loop integrals. Zero-temperature-type integrals were defined by analytic continuation while integrals containing a BoseEinstein factor were cut off by an infrared regulator. In the examples considered the prescriptions allowed one to extrapolate gauge-invariant quantities calculated near $D=D_{0}=4$ down to $D=2$ in the required manner. Instead of the two prescriptions, one might try the following single condition : analytically continued gauge invariant quantities in pure $Y M$ theory should be nondiverging at $D=2$. A relook at the examples shows that this also provides a nontrivial check. In the absence of an a priori justification of the prescriptions, one is actually checking both the IR prescriptions and the gauge-invariance. Still, the analysis of gauge-invariant structures for a variable $D$ appears instructive and one might want to consider more examples and at higher order. It might also be interesting to explore the $D \rightarrow 2$ procedure for gauge-invariant quantities correctly evaluated near $D_{0}=3$ [15, 16] .

Fermions can be accomodated by using the number of flavours, $N_{f}$, as a parameter. The $N_{f} \neq 0$ part of any gauge-invariant quantity must be invariant by itself. At low orders in perturbation theory, one may even entertain the notion of calculating the $N_{f}=0$ and $N_{f} \neq 0$ sectors with different gauge-fixing. For example, the pure glue part can be calculated in the Feynman- $D$ gauge while the $N_{f} \neq 0$ part can be calculated in the $\alpha$-gauge to check gauge-invariance. Whether such hybrid calculations are useful or practical should be decided on a case by case basis. Likewise, scalars can be coupled by taking $N_{s}$ copies of them.

Finally some comment on the background field gauge [29]. This is one way of calculating in quantum field theory while keeping classical gauge invariance at every step. The gauge-invariance here is with respect to the background field $B_{\mu}$ which is introduced for this purpose and gives no information about the physical gaugeinvariance of any quantity calculated. In particular, the quantum part of the action must still be gauge fixed. Thus even here one might use the $D$ parameter without redundancy. 


\section{Acknowledgements}

I thank J.P. Blaizot, C. Corianó, A.S. Goldhaber, E. Iancu, H. Osborn, R.D. Pisarski and J.C. Taylor for very helpful discussions. I also acknowledge stimulating and hospitable visits to DAMTP-Cambridge and Martignano-Italy during the course of this work.

\section{Appendix}

1.Some formulae are collected here for ease of reference.

a) The bosonic $\left(q_{0}=2 \pi n T\right)$ sums needed in Sect.(3) are [8]

$$
\begin{aligned}
& T \sum_{q_{0}} \frac{1}{Q^{2}}=\frac{n(q)}{q}+\frac{1}{2 q}, \\
& T \sum_{q_{0}} \frac{1}{Q^{4}}=\frac{n(q)}{2 q^{3}}-\frac{1}{2 q^{2}} \frac{d n(q)}{d q}+\frac{1}{4 q^{3}},
\end{aligned}
$$

where $n(q)=(\exp \beta q-1)^{-1}$ is the Bose-Einstein factor. The last terms in the above sums are temperature independent and drop when dimensional regularisation is used for the $\vec{q}$-integrals .

b) The angular integrals for $(D-1)$ dimensional Euclidean space have been defined by 4

$$
\omega(D)=\int \frac{d \Omega_{D-1}}{(2 \pi)^{(D-1)}} \equiv\left[2^{(D-2)} \Gamma\left(\frac{D-1}{2}\right) \pi^{\frac{(D-1)}{2}}\right]^{-1} .
$$

c)The zero temperature integrals in Sects.(3,5) are evaluated using [1, 2, 3, 4],

$$
\begin{aligned}
\int \frac{d^{s} q}{(2 \pi)^{s}} \frac{1}{Q^{2}(Q+K)^{2}} & =(4 \pi)^{-\frac{s}{2}}\left(K^{2}\right)^{\frac{(s-4)}{2}} \frac{\Gamma\left(2-\frac{s}{2}\right) \Gamma^{2}\left(\frac{s}{2}-1\right)}{\Gamma(s-2)}, \\
\int \frac{d^{s} q}{(2 \pi)^{s}} \frac{1}{Q^{2}+M^{2}} & =(4 \pi)^{-\frac{s}{2}}\left(M^{2}\right)^{\frac{(s-2)}{2}} \Gamma\left(1-\frac{s}{2}\right) .
\end{aligned}
$$

d)Expressions containing gamma-functions can be simplified with the following very useful identities [14

$$
\begin{aligned}
\Gamma(1+z) & =z \Gamma(z), \\
\Gamma(z) \Gamma(1-z) & =\frac{\pi}{\sin \pi z},
\end{aligned}
$$




$$
\sqrt{\pi} \Gamma(2 z)=2^{(2 z-1)} \Gamma(z) \Gamma\left(z+\frac{1}{2}\right)
$$

2. The one-loop gluon self energy is given by

$$
\Pi_{\mu \nu}^{a b}(K)=\int[d q]\left\{L_{\mu \nu}^{a b}(K, Q)+\frac{1}{2} M_{\mu \nu}^{a b}(K, Q)+\frac{1}{2} N_{\mu \nu}^{a b}(K, Q)\right\}
$$

where $(\mu \nu)$ are the Lorentz indices and $(a b)$ the group indices. The symmetry factors have been explicitly displayed. $L$ is the ghost loop contribution ( -1 factor included), $M$ the tadpole diagram and $N$ is due to the tri-gluon coupling. Expressions for $L, M$ and $N$ in $D$ dimensions may be found, for example, in [4]. The complete result at zero temperature, in an $\alpha$-covariant gauge, with the integrals done, may also be found in [4]. For the Landau gauge $(\alpha=-1)$ the expression is contained in Ref. 27 which studies $Q C D$ in $2+\epsilon(\epsilon \ll 1)$ dimensions and also notes the divergence of the selfenergy as $\epsilon \rightarrow 0$. 
3. The Free energy.

a)The contribution of each massless, bosonic degree of freedom to the ideal gas pressure is

$$
\begin{aligned}
P_{0}^{b} & =-\frac{1}{2 V \beta} \ln \operatorname{Det}\left(-\partial^{2}\right) \\
& =-\frac{1}{2} \int[d q] \ln \left(Q^{2}\right) \\
& =-T \int \frac{d^{(D-1)} q}{(2 \pi)^{(D-1)}} \ln \left(1-e^{-\beta q}\right) \\
& =-T^{D} \omega(D) \int_{0}^{\infty} d x x^{(D-2)} \ln \left(1-e^{-x}\right) \\
& =T^{D} \omega(D) \sum_{p=1}^{\infty} \frac{1}{p} \int_{0}^{\infty} d x x^{(D-2)} e^{-p x} \\
& =T^{D} \omega(D) \Gamma(D-1) \zeta(D) \\
& =T^{D} \pi^{-\frac{D}{2}} \zeta(D) \Gamma(D / 2) .
\end{aligned}
$$

The determinant above is evaluated with the required periodic boundary conditions [7, 8]. In the second line a $T$-independent piece was dropped. The interchange, in order, of the integration and power series summation is justified for $D>1$. Final simplification is achieved using the definition of the gamma and zeta functions [14 and the use of formulae in Note(1) of this appendix. In passing it is noted that for massless fermions at zero chemical potential, each modes contribution to the ideal pressure will turn out to be eq. A.16) multiplied by a statistical factor $\left(1-2^{(1-D)}\right)$. For the case of massive particles, nonzero chemical potentials and background fields, see 26].

b)For the calculation of the order $g^{2}$ contribution to the pressure, one can save some effort and reduce errors by proceeding as follows

$$
P_{2}=\int[d k] \int[d q] D(K)\left\{\frac{1}{2} L+\frac{1}{8} M+\frac{1}{12} N\right\} .
$$

That is, compute the expression in curly brackets first. Here $D(K)=\frac{\delta^{a b}}{K^{2}}(\delta \mu \nu+$ $\left.\alpha \frac{K^{\mu} K^{\nu}}{K^{2}}\right)$ is the free propagator in the $\alpha$-covariant gauge and $L, M$ and $N$ are the $\alpha$ dependent tensors used in eq.(A.9) above. The $\alpha$ dependent pieces of $P_{2}$ cancel 
only after frequent use of the identity $2 K . Q=(K+Q)^{2}-K^{2}-Q^{2}$, changes of sum-integration variables, and shifts of sum-integration variables (assumed valid), to obtain the final answer displayed in (5.4). Expressions similar to (5.4) in the background Feynman gauge may be found in [28].

c)The plasmon contribution in four dimensions has been calculated by Kapusta 24] in the Feynman gauge. Here I sketch the $D$ dimensional analog in the Coulomb gauge, using the notation of Toimela [25]. One begins with

$$
\begin{aligned}
P_{\text {plas }} & =\frac{1}{2 \beta} \int[d q] \sum_{p=2}^{\infty} \frac{1}{p} \operatorname{Tr}\left(-D^{c} \Pi\right)^{p} \\
& =\frac{\left(N_{c}^{2}-1\right)}{2 \beta} \int[d q] \sum_{p=2}^{\infty} \frac{(-1)^{p}}{p}\left[\left(\frac{F}{q^{2}}\right)^{p}+(D-2)\left(\frac{G}{Q^{2}}\right)^{p}\right]
\end{aligned}
$$

In the above $D^{c}$ is the free propagator in the (strict) Coulomb gauge

$$
D_{\mu \nu}^{c}=\frac{\delta_{\mu 0} \delta_{\nu 0}}{k^{2}}+\frac{\delta_{\mu i} \delta_{\nu j}}{K^{2}}\left(\delta_{i j}-\frac{k_{i} k_{j}}{k^{2}}\right),
$$

$\Pi$ is the one-loop self energy, $F \equiv \Pi_{00}$, and $G$ is the transverse part of $\Pi_{i j}$ :

$(D-2) G=\Pi_{i j}\left(\delta_{i j}-q_{i} q_{j} / q^{2}\right)$, with sum over repeated indices.

In order to obtain the leading plasmon-like $\left(>g^{4}\right)$ contribution from $P_{\text {plas }}$, one need only look at the infrared region which lies in $q_{0}$ sector. Now, in four dimensions we have $F \sim g^{2} T^{2}$ and $G \sim g^{2} k T$. In $D$ dimensions near $D=4$ one therefore expects $F \sim g^{2} T^{(D-2)}$ and $G \sim g^{2} T k^{(D-3)}$. Using the dimensionless coupling $g_{0}$ defined by $g^{2}=g_{0}^{2} T^{(4-D)}$, and assuming $g_{0} \ll 1$, consider the contribution of soft $\left(\sim g_{0} T\right)$ loop momenta to the $p$-th term in $P_{\text {plas }}$. The electric ( $F$ type) contribution will be

$$
\sim T\left(\frac{g_{0}^{2} T^{(4-D)} T^{(D-2)}}{\left(g_{0} T\right)^{2}}\right)^{p}\left(g_{0} T\right)^{(D-1)}=T^{D} g_{0}^{(D-1)}
$$

while the magnetic ( $G$ type) contribution is

$$
\sim T\left(\frac{g_{0}^{2} T^{(4-D)} T\left(g_{0} T\right)^{(D-3)}}{\left(g_{0} T\right)^{2}}\right)^{p}\left(g_{0} T\right)^{(D-1)}=T^{D} g_{0}^{(D-1)+(D-3) p} .
$$

The electric contribution is plasmon like for all $p$ and for $D<5$. When $D>3$, the magnetic contribution is plasmon-like only for $p<(5-D) /(D-3)$. Also since $p \geq 2$, 
this implies $D<11 / 3$. Thus for $3<D<11 / 3$, only the finite number of terms, $2 \leq p<(5-D) /(D-3)$, give a plasmon-like contribution in the magnetic sector. The magnetic contribution might also be plasmon-like for $D \leq 3$. On the other hand, it is easy to see from the equations that for $D>3$, the magnetic contribution is always subleading to the electric contribution. Thus the leading plasmon contribution for $D>3$ is given by eq. (5.7) of the main text. The integral may be evaluated by the formulae listed in Note(1) of this appendix : The second term in (5.7) drops in dimensional regularisation while the logarithm is integrated by considering first its derivative with respect to $m_{e l}^{2}$. It is amusing to note that the peculiar ratio $11 / 3$ appearing in the above analysis occurs in a natural but apparently unrelated way also in the beta function.

\section{Beta Function}

The beta function is easiest to calculate by using background field techniques [29]. One first computes (see Abbott [29] for details and further references) the wavefunction renormalisation factor $Z_{B}$ of the background field $B_{\mu}$ obtained from its selfenergy. In the background Feynman gauge one can obtain

$$
\Pi_{(\mu \nu),(a b)}^{(B)}(K)=-g^{2} N_{c} \delta_{a b}\left(K^{2} g_{\mu \nu}-K_{\mu} K_{\nu}\right) \frac{(7 D-6)}{(2 D-2)} \int \frac{d^{D} q}{(2 \pi)^{D}} \frac{1}{Q^{2}(Q+K)^{2}} .
$$

Like the usual self-energy (A.9), this self-energy (A.23) is not gauge-invariant. Using formulae given in the beginning of this appendix one may check that this background field self energy also diverges as $D \rightarrow 2$. The gauge-invariant information in (A.23) comes from the residue, $Z_{B}^{(1)}$, of the $\epsilon=(4-D) / 2$ pole in $Z_{B}$, when the integrals are computed in dimensional regularisation. The beta function is given by $\beta(g)=$ $-\frac{1}{2} g^{2} \frac{\partial Z_{B}^{(1)}}{\partial g}$. The $D$ dependent terms outside the integrals in (A.23) give the famous $11 / 3$ factor. 


\section{References}

[1] C. G. Bollini and J. J. Giambiagi, Phys. Lett. B 40(1972) 566; Nuovo Cim. B 12 (1972) 20;

G. 't Hooft and M. Veltman, Nucl. Phys. B 44 (1972) 189;

J. F. Ashmore, Lett. Nuovo Cim. 4 (1972) 289;

G. M. Cicuta and E. Montaldi, Lett. Nuovo Cim. 4 (1972) 329.

[2] R. Gastmans and R. Meuldermans, Nucl. Phys. B 63 (1973) 277;

W. J. Marciano, Phys. Rev. D 12 (1975) 3861.

[3] J. C. Collins, Renormalization (Cambridge University Press, England, 1984).

[4] T. Muta, Foundations of Quantum Chromodynamics (World Scientific, Singapore 1987).

[5] W. Siegel, Nucl. Phys. B 156 (1979) 135;

J. F. Schonfeld, Nucl. Phys. B 185 (1981) 157;

S. Deser, R. Jackiw and S. Templeton, Ann. Phys. (NY) 140 (1982) 372.

[6] G. 't Hooft, Nucl. Phys. B 75 (1974) 461.

[7] C. W. Bernard, Phys. Rev. D 9 (1974) 3312.

[8] D. J. Gross, R. D. Pisarski and L.G. Yaffe, Rev. Mod. Phys. 53 (1981) 43;

N. P. Landsman and Ch. G. van Weert, Phys. Rep. 145 (1987) 141;

J. Kapusta, Finite Temperature Field Theory (Cambridge University Press, England, 1985).

[9] B. L. Combridge, J. Kripfganz and J. Ranft, Phys. Lett. B 70 (1977) 234;

R. Cutler and D. Sivers, Phys. Rev. D 17 (1977) 196;

J. F. Owens, E. Reya and M. Glück, Phys. Rev. D 18 (1978) 1501.

[10] R. K. Ellis and J. C. Sexton, Nucl. Phys. B 269 (1986) 445.

[11] S. Nadkarni, Phys. Rev. D 33 (1986) 3738;

T. Toimela, Z. Phys. C 27 (1985) 289.

[12] T. Toimela, Phys. Letts. B 124 (1983) 407 and Int. J. Theor. Phys. 24 (1985) 901 ;

S. Nadkarni, Phys. Rev. D 27 (1983) 917.

U. Heinz, K. Kajantie and T. Toimela, Ann. Phys. (NY) 176 (1987) 218.

[13] O. Kalashnikov and V. Klimov, Sov. J. Nucl. Phys. 31 (1980) 699.

[14] I. S. Gradshteyn and I. M. Ryzhik, Tables of Integrals, Series and Products (Academic Press, NY 1980).

[15] E. D' Hoker, Nucl. Phys. B 201 (1982) 401.

[16] R. Jackiw and S. Templeton, Phys. Rev. D 23 (1981) 2291;

T. Appelquist and R. D. Pisarski, Phys. Rev. D 23 (1981) 2305. 
[17] P. V. Landshoff and A. Rebhan, Nucl. Phys. B 383 (1992) 607.

[18] R. D. Pisarski, Phys. Rev. Letts. 63 (1989) 1129;

E. Braaten and R. D. Pisarski, Nucl. Phys. B 337 (1990) 569 and Phys. Rev. D 42 (1990) R2156.

[19] J. Frenkel and J. C. Taylor, Nucl. Phys. B 334 (1990) 199.

[20] R. Kobes, G. Kunstatter and A. Rebhan, Phys. Rev. Letts. 64 (1990) 2992 and Nucl. Phys. B 355 (1991) 1.

[21] J. C. Taylor and S. M. H. Wong, Nucl. Phys. B 346 (1990) 115;

E. Braaten and R. D. Pisarski, Phys. Rev. D 45 (1992) R1827;

J. Frenkel and J. C. Taylor, Nucl. Phys. B 374 (1992) 156;

R. Efraty and V. P. Nair, Phys. Rev. Letts. 68 (1992) 2891.

[22] J. P. Blaizot and E. Iancu, Nucl. Phys. B 390 (1993) 589, and Saclay preprint SPhT/93-01 (to appear in Phys. Rev. Letts.).

[23] V. V. Klimov, Sov. Phys. JETP 55 (1982) 199;

H. A. Weldon, Phys. Rev. D 26 (1982) 1394.

[24] J. Kapusta, Nucl. Phys. B 148 (1979) 461.

[25] T. Toimela, Z. Phys. C 17 (1983) 365.

[26] H. A. Weldon, Nucl. Phys. B 270 (1986) 79;

A. Actor, Forstschr. Phys. 35 (1987) 793.

[27] M. Dine, C. Litwin and L. McLerran, Phys. Rev. D 23 (1981) 451.

[28] K. Shiraishi and S. Hirenzaki, Z. Phys. C 53 (1992) 91.

[29] B. S. DeWitt, Dynamical Theory of Groups and Fields (Gordon and Breach, New York 1965);

L. F. Abbott, Nucl. Phys. B 185 (1981) 189. 